\title{
A catalog of the types of Heteroptera (Insecta) deposited in the Museo Argentino de Ciencias Naturales, Buenos Aires 2. Addenda et corrigendum
}

\author{
Axel O. BACHMANN
}

División Entomología, Museo Argentino de Ciencias Naturales, Av. A. Gallardo 470, Buenos Aires, C1405DJR. Departamento de Biodiversidad y Biología Experimental, Facultad de Ciencias Exactas y Naturales, Universidad de Buenos Aires, C1428EHA, Argentina. e-mail: bachmann@bg.fcen.uba.ar

\begin{abstract}
After the publication of the first part of this catalog (Bachmann 1999), several type specimens were deposited, or found in the collection, and are here recorded. Some entries correspond to additional specimens of species already dealt with. In these addenda types of 69 names are recorded. A correction of the authors' names of one previous entry is included (Pothea andina, Reduviidae).
\end{abstract}

Key words: Insecta, Hemiptera, Heteroptera, Type specimens-2.

Resumen: Catálogo de los tipos de Heteroptera (Insecta) conservados en el Museo Argentino de Ciencias Naturales, Buenos Aires. 2. Addenda et corrigendum. Después de la publicación de la primera parte de este catálogo (Bachmann 1999), varios ejemplares tipo fueron depositados, o hallados en la colección, y son registrados aquí. Algunos registros corresponden a ejemplares adicionales de especies ya tratadas. En estos addenda se registran tipos de 69 nombres. Se incluye una corrección de los nombres de los autores de un registro anterior (Pothea andina, Reduviidae).

Palabras clave: Insecta, Hemiptera, Heteroptera, Ejemplares tipo-2.

\section{INTRODUCTION}

A catalog of the types of Heteroptera deposited in this Museum was published several years ago (Bachmann, 1999); thereafter further types were here deposited, and some others were found in the collection, not included then. Sixty-nine names are recorded in this addendum. Many of the new recorded types refer to twenty-one species of Reduviidae described by Dr. Diego José Carpintero, and to sixteen species of Miridae described by Dr. Jose Candido de Melo Carvalho and Mr. Diego Leonardo Carpintero; both series were here deposited by Diego L. Carpintero, after the decease of his father, Dr. Diego J. Carpintero.

As before, the specific and subspecific names are alphabetically arranged in separate lists, grouped by families, in their original spellings, with a brief bibliographic quotation, and, if they were mentioned, the localities and specimens designated. A list of the types actually housed in the Museum follows, with a description of all the labels. If no holotype, or an equivalent was origi- nally designated, and the amount of specimens examined was not stated, it is assumed that there was a series of syntypes (recommendation $73 \mathrm{~F}$ of the Code, ICZN, 1999), eventually 'sole syntypes'.

Burmeister did not use to label his specimens; he fastened to the bottom of the drawer, to the left of the specimens, handwritten identification labels, genus and/or species, adding sometimes a vague distributional indication, e. g. "Patag.", "Arg." or "Br." for Patagonia, Argentina or Brasil; types were not identified. Berg put to his types a tiny label, printed "Typus" in red and red framed. I added to the specimens deemed to be the types, labels reading "Holotypus" or "Syntypus", printed or handwritten on red paper. On the other hand, there are in the collection some specimens labeled as types, but not mentioned in the original descriptions; some were perhaps not even intended as types, but in order to assist in future research they are here included in a separate list. If the country is not mentioned, Argentina is meant.

Some specimens bear a registration number, 
handwritten on a small label with a red frame, entered in the register book of the Entomology Division; numbers under 10000 refer to the old general register books of the Museum.

This Museum is identified as MACN. Other repositories are identified as follows: AMNH, American Museum of Natural History, New York, USA; BM, Natural History Museum, London, UK; CAM, Carnegie Museum, USA; CAS, California Academy of Sciences, USA; CC, Dr. D.J. Carpintero coll.; CJC, J.C.M. Carvalho coll.; CM , A. Martínez coll.; CNC, Canadian National Collection; COU, Cornell University, USA; CRC, R.U. Carcavallo coll.; DL, D.L. Carpintero coll.; HIP, Hydrobiologisches Institut, Plön, Germany; IBH, Instituto de Ciências Biologicas, Belo Horizonte, Brasil; IBM, Instituto de Biología, Universidad Nacional Autónoma, México; IML, Fundación e Instituto Miguel Lillo, Tucumán; ISM, Instituto San Miguel, San Miguel, later INESALT, now at the Universidad Nacional de Salta, Salta; KU, Kansas University, USA; MLE, Museum Leningrad, Russia; MLP, Museo de La Plata, La Plata; MO, Museum Oxford, UK; MRJ, Museu Nacional, Rio de Janeiro, Brasil; MS, Museum Stockholm, Sweden; RL, Rijsmuseum Leiden, The Netherlands; SEM, Snow Entomological Museum, Lawrence, USA; UM, University of Minnesota, USA; USNM, United States National Museum (Smithsonian Institution), Washington, USA; ZMU, Zoologisch Museum, Utrecht, The Netherlands.

Abbreviations used: ab., aberratio; allot., allotypus, allotype; coll., collection; f., figure; genit., genitalia; handwr., handwritten; holot., holotypus, holotype; leg., legit, legint; p., page; parat., paratypus, paratype; pl., plate; print., printed; prov., province; spec., specimen, specimens; synt., syntypus, syntype; tab., table; xerocop., xerocopied.

\section{CATALOG OF THE TYPES}

\section{Belostomatidae}

bordoni [Belostoma]. De Carlo 1966: 106-107, f. 8, 28, 32. Venezuela: Guárico: represa del Calabozo, leg. Bordon \& Martínez VI.1963. Holot. $\hat{\sigma}$, alot. + , parat. four $\hat{\sigma}$, one $q$ in MACN.

Holot. $\partial$, allot. + , three parat. pinned, "Venezuela / Est. de Guarico / Leg. Bordon Martínez / VI - 1963" handwr., "Holotypus" ["Allotypus", "Paratypus"] print. on red [red, yellowish orange] paper, black frame; "Belostoma / bordoni / Holotipo [Alotipo, Paratipo] [handwr.] / Museo Argentino de Ciencias Naturales [print.]" on red [red, white] paper, black frame.

Note: Two parat. were recorded in the main catalog (Bachmann 1999: 194).

doesburgi [Belostoma]. De Carlo 1966: 104-105, f. 24, 29. Surinam: Paramaribo, leg. Doesburg IX.1962. Holot. $\widehat{c}$, allot. + , six parat. in RL, six parat. 53994 in MACN.

One parat. pinned, "Paramaribo / Surinam / 15-IX-1962 / P.H. v. Doesburg" print., date handwr.; "Paratypus / Museo Argentino de Ciencias Naturales" print. on yellowish orange paper.

Notes: Eight parat. were recorded in the main catalog (Bachmann 1999: 194). A synonym of $B$. harrisi Lauck 1962, fide Nieser (1975: 99-100).

sattleri [Belostoma]. De Carlo 1966: 105-106, f. 9, 12, 13. Brasil: Amazonas, holot. $\delta^{\lambda}$, allot. $\bigcirc$ in HIP, one $q$ parat. in MACN sub 53995.

Holot. of pinned, dissected parts in a plastic microvial, "Amazonasgebiet / Bragantina / Quatipuru / 6-4-69 / leg, E.J. Fittkau / coll. Amazonas 1960/3 [print.] / A 519 [handwr.]"; "Belostoma / harrisi Lauck / Det.: D.R. Lauck" print.; "Holotypus / Museo Argentino de Ciencias Naturales" print. on red paper, black frame.

Notes: The allot. $\bigcirc$ was recorded in the main catalog (Bachmann 1999: 196). A synonym of $B$. harrisi Lauck 1962, as labeled by Lauck.

schubarti [Horvathinia]. De Carlo 1958: 5051, f. 9, 16, 18, 26. Brasil: Pirassununga, leg. Schubart 18.XI.1940, Holot. $\delta$ in MACN sub 53801.

A glass vial with genitalia dissected from the holot., "Horvathinia / schubarti / genitales" handwr. by De Carlo on white, dirty paper.

Notes: The holot. 0 , but not its genit., was recorded in the main catalog (Bachmann 1999: 196). A synonym of $H$. pelocoroides Montandon 1911 fide Schnack \& Estévez (2005).

\section{Cimicidae}

patagonicus [Cyanolicimex]. Carpintero, Di Iorio, Turienzo \& Masello in Di Iorio, Turienzo, Masello \& Carpintero 2010: 6-7, f. 2-7, 27. Holot. $\uparrow$, allot. $\hat{O}$, parat. three $\odot$, one $\delta$, nymphs II, IV, V, all slide mounted, Rio Negro Prov.: El Cóndor, in nests of Cyanoliseus patagonus (Aves: Psittacidae).

Holot. $q$ in a slide, cleared and mounted in toto, "Arg. Rio Negro / El Condor / XII.2004 / J. 
Masello / on Cyanoliseus / nest / Cyanolicimex / patagonicus n. sp." handwr.; "Holotypus" print. on red paper, remainings of a black frame; "Cyanolicimex / patagonicus n. sp. / Carpintero, Di Iorio, Turienzo / \& Masello [handwr.] / Museo Argentino de Ciencias Naturales [print.]" on red paper, remainings of a black frame.

\section{Coreidae}

ambiguus [Scamurius]. Brailovsky 1986: 30, 38-39, f. 49, 52. Misiones Prov.: Loreto III.1953. Holot. $\delta$ in MACN; parat. in IBM.

Holot. $\delta$ pinned, dissected parts on a card, "Holotipo" print. on bright red paper, black frame, reverse white; "Loreto / Misiones / III1953" handwr., remainings of a black frame; " $\mathrm{H}$. Brailovsky A. det. [print.] / Scamurius ambiguus / Brailovsky [handwr.]”, black frame.

cauta [Cebrenis]. Brailovsky 1995: 31, 75-83, f. 30, 31, 62-65, 111, 112, 187. Corrientes Prov.: 32 $\mathrm{km}$ East of Ituzaingó 20.I.1989. Holot. in IBM. Three parat.: Misiones Prov. 18.VIII.1951. Two parat: Misiones Prov. 2-4.III.1897 coll. Venturi. Two parat: Catamarca Prov. One parat: Corrientes Prov.: Santo Tomé II.1926, all in MACN. Several parat. from West Indies southwards until Argentina, in diverse repositories.

Three parat. pinned, " 5653 " handwr., red frame; "Rca. Argentina / Gob. Misiones / 2.III.1897 [3.III.1897, 4.III.1897] / S. Venturi" print., black frame. Two parat.: "10214" handwr., red frame; "Catamarca" handwr. on green paper, black frame, reverse white. One parat.: "S. Tomé / Corrientes [print.] / II.926 [handwr.]", remainings of a black frame. All six: "Paratipo" print. on bright red paper, black frame; "H. Brailovsky A. det. [print.] / Cebrenis / cauta Brailovsky [handwr.]", black frame.

Note: The enter 5653 in the old register book of the Museum refers to 2127 hemipters sold by S. Venturi, and entered on 17.IX.1901; the entry 10214 in the register book of the Entomology Division refers to 52 hemipters from Catamarca Prov., entered on XII.1910.

marianae [Scamurius]. Brailovsky 1986: 30, 42-45, f. 51. Paraguay: Horqueta, East of Paraguay River. Holot. $\delta$ in AMNH, Corrientes Prov.: Santo Tomé II.1925 one parat. $\delta$ in MACN. Other parat. Brasil in MRJ and IBM; Catamarca Prov.: La Viña in IBM.

One parat. $\hat{\alpha}$ pinned, "S. Tomé / Corrientes [print.]/II.925 [handwr.]" black frame; "Paratipo" handwr. on green paper; "H. Brailovsky A. det. [print.] / Scamurius marianae / Brailovsky [handwr.]" on white paper, black frame.

mundulus [Scamurius]. Brailovsky 1986: 30-32, f. 50, 54. Corrientes Prov.: Santo Tomé I.1928 holot. ${ }^{\top}$, allot. $\circ$ in MACN. Paraguay: Villa Rica 12.II.1947, leg. Schade, one parat. $\partial$ in IBM.

Holot. Ô, one parat. pinned, "S. Tomé / Corrientes [print.] / I.928 [handwr.]", black frame; "H. Brailovsky A. det. [print.] / Scamurius mundulus / Brailovsky [handwr.]", black frame; "Holotipo" ["Paratipo"] print. on bright red [bright green] paper.

serus [Pachylis]. Berg 1881: 260-261. Brasil: Rio

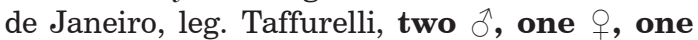
"pseudoimagen". There are some spec., not identified, in Berlin Museum.

One $\hat{d}$, one + , one nymph synt. pinned, "Typus" print. with red ink on tiny labels, red frame; "Rio de / Jan." print. on green paper. "Pachylis" and "serus / Berg / Br." handwr. by Berg, black frame, originally fastened to the bottom of the drawer. To all I add "Pachylis / serus / Berg / Syntypus" handwr. on red paper, remainings of a black frame.

vacillans [Scamurius]. Brailovsky 1986: 30, 4142, f. 44. Paraguay: Horqueta, East of Paraguay River 14.IV.1933, leg. Schulze, holot. in AMNH. Parat.: Corrientes Prov.: Santo Tomé II.1925; Catamarca Prov.; Brasil: Rio de Janeiro, in IBM, MRJ.

One parat. pinned, dissected parts on a card, "Buenos Aires Argentina / Isla Martín García / 4-1938 M.J. Viana" print., date handwr., "42533" handwr., remainings of a red frame; "Paratipo" print. on bright green paper, black frame; "H. Brailovsky A. det. [print.] / Scamurius vacillans / Brailovsky [handwr.]", black frame.

Notes: Martín García Island, in the Rio de la Plata, was not mentioned in the original publication, but the labels are authentic as from Brailovsky. The entry 42533 in the register book of the Entomology Division refers to one coreid from Martín García Is., M.J. Viana leg., designated parat. of Scamurius vacillans.

\section{Gelastocoridae}

decarloi [Gelastocoris]. Estévez \& Schnack 1977: 81-83, f. 1-8. Brasil: Santa Catarina: Nova Teutonia, holot., allot., four parat. in MACN. 
Holot., allot., four parat. pinned [one of the parat. on a card, with a further card beneath], "Brasilien / Nova Teutonia / $27^{\circ} 11$ B $52^{\circ} 23$ L / Fritz Plaumann / 300-500 m [print.] / [diverse dates, handwr.]", black frame; "Museo de La Plata [print.] / Gelastocoris / decarloi / Estévez - Schnack [handwr.]", black frame; "Holotipo" ["Alotipo", "Paratipo"] print. on red paper.

\section{Gerridae}

pingreensis [Gerris]. Drake \& Hottes 1925: 49. United States: Colorado: Pingree Park.

One parat. pinned, "Pingree Park / Colo [print.] 8-22-24 [handwr.]"; "paratype" print. on pink paper; "D.J. Drake / Collector" print.; "F.C. Hottes" print.; " 46866 " handwr., remainings of a red frame; "Gerris / pingreensis / D+Hot / D+H" handwr., black frame.

\section{Hydrometridae}

quadrispina [Hydrometra]. Perez Goodwyn 2001: 127-128, f. 1-8. Buenos Aires Prov.: Ensenada: Punta Lara XII.1997 - II.1998, holot. $\bigcirc$ (sic, pro $\overbrace{}^{\Uparrow})$, allot. $\odot$, six parat. in MLP. Parat. two ${ }^{\circ}$, four $\circ$ in MACN.

Two ${ }^{2}$, four + parat. on big cards with a layer of cotton, wrapped in polyethylene film, on separate pins, "Argentina, Buenos / Aires, Punta Lara / 19/12/97 [on one] 29/1/98 [on five] / col. N. von Ellenrieder [on one] col. P. Goodwyn [on five]" handwr., black frame; "Hydrometra / quadrispina / Perez Goodwyn / Paratipo" handwr., black frame; "Paratipo" print. on red paper.

wileyi [Hydrometra]. Hungerford 1923: 57-58, pl. I: f. 1, 6, 9-11. United States of America: Texas: Colorado County, near Rock Island, holot., allot., parat. in $\mathrm{KU}$; other parat. in USNM, CAM, CNC, CU, UM and several private collections.

One parat. on a pointed card, head and parts of legs missing, "Colorado Co. Texas / July 22. 1922 / Mrs. Grace Wiley" photographed; "Hydrometra wileyi / Paratype [handwr.] / Det. H.B. Hungerford [print.]" on pink paper; "1850" handwr.

\section{Largidae}

nigricans [Thaumastaneis]. Dellapé \& Melo 2007: 22-24, f. 1, 7-11. Bolivia; Trinidad 50276, "montandoni det. Kormilev", holot. $\sigma^{\lambda}$ in MACN. Bolivia: Santa Cruz: Santiago: Chiquitos, parat. $\odot$ in IML.
Holot. on a pointed card, dissected parts on another pointed card, a plastic microvial with dissected parts; "50276" handwr., red frame; “” print.; "Trinidad / Bolivia / X.1917 [print., obverse] / J. Bosq leg. [handwr., reverse]"; "Holotipo" handwr. on red paper; "Taumastaneis (sic) / montandoni / Kirkaldi (sic) \& Edwards / det. N. Kormilev [handwr.] / Museo Argentino de Ciencias Naturales [print.]" on white paper, black frame; "Thaumastaneis / nigricans / Dellapé \& Melo 2007" handwr. on white paper.

Note: The entry 50276 in the register book of the Entomology Division refers to one "pyrrhocorid" from Bolivia: Trinidad, leg. C. Lizer y Trelles X.1917, labeled Taumastaneis montandoni by N. Kormilev.

podalicus [Acinocoris]. Brailovsky 1989: 78, f. 6. Formosa Prov.: Formosa City XII.1949. Holot in MACN.

Holot. $q$ on a pointed card, "Holotipo" print. on bright red paper, black frame; "Argentina / Formosa / Ciudad XII.1949 / A. Martínez legit" print., black frame; "H. Brailovsky A. det. [print.] / Acinocoris / podalicus Brailovsky [handwr.]" on white paper, black frame.

\section{Miridae}

argentina [Hadronemisca]. Carvalho \& Wallerstein 1978: 523-524, f. 1-5. San Luis Prov.: San Gerónimo 1972, holot. ô in MACN. San Luis Prov.: S. Gerónimo and Merlo, parat. three $\delta$ in MACN and CJC.

Holot. $\delta$ on a card, "S. Jeronimo / San Luis / 1972" handwr.; "Holotypus" print. on red paper, black frame; "17" typed; "Hadronemisca / argentina / n. sp. [handwr.] / Det. J.C.M. Carvalho [print.]" on white paper.

Note: Parat. not found.

cajamarcanus [Mimoncopeltis]. Carvalho \& D.L. Carpintero 1989: 1116, f. 22-26. Perú: Cajamarca: Cajamarca, 2700 m, III.1942, holot. ô in DL.

Holot. $\delta$ on a card, a leg on the same card, dissected parts on another card, "Cajamarca / Peru III.42 / 2700 m" handwr.; "1294" handwr. with green ink; "Holotypus" print. on red paper, remainings of a black frame; "Mimoncopeltus / cajamarcanus n. sp. [handwr.] / J.C.M. Carvalho det. 88 [print.]" on white paper, black frame.

carpinteroi [Polymerus]. Carvalho \& Wallerstein 1978: 526-527, f. 14-17. San Luis Prov.: Merlo 
1972, holot. $\delta$ in MACN.

Holot. $\delta$ on a card, dissected parts on another card, "Merlo / S. Luis / 1972" handwr.; "Holotipo" print. on dark red paper, black frame; "Polymerus / carpinteroi / n. sp. [handwr.] / Det. J.C.M. Carvalho [print.]" on white paper; " 26 " typed.

chaquensis [Chiloxionotus]. Carvalho \& D.L. Carpintero 1989: 1110-1113, f. 6-9. Chaco Prov.: Resistencia XII.1935, holot. $\delta$ in DL.

Holot. $\delta$ on a pointed card, dissected parts on another card, "Resistencia / Chaco / XII-35" handwr.; "1487" handwr. with green ink; "Holotypus" print. on red paper; "Chiloxionotus / chaquensis n. sp. [handwr.] / C.\& C. / J.M. Carvalho det. 1988 [print.]" on white paper.

chingonus [Comefulvius]. Carvalho \& D.L. Carpintero 1985: 510-511, f. 6, 7. Córdoba Prov.: La Serranita II.1985, holot. $\delta$ in DL.

Holot. $\delta$ on a pointed card, a leg on the same card, "La Serranita / Cordoba / II-1981" handwr.; "56" typed with red ink; "Holotypus" print. on red paper; "Comefulvius / chingonus n. sp. / [handwr.] / det. J.C.M. Carvalho 19_ [print.]" on white paper, black frame.

cordobensis [Nanniresthenia]. Carvalho \& D.L. Carpintero 1987: 20-23, f. 5-9. Córdoba Prov.: Calamuchita I.1978, holot. $\delta$ in DL.

Holot. $\delta$ on a pointed card, dissected parts on another card, "Calamuchita / Córdoba" handwr.; "Holotypus" print. on red paper, black frame; "Nanniresthenia / cordobensis / n. sp. / Det. Carpintero" handwr., black frame; "1278" handwr. with green ink.

dureti [Platytylus]. Carvalho \& D.L. Carpintero 1989: 1119, f. 20. Misiones Prov.: Iguazú, leg. Duret XI.1945, holot. $\widehat{\delta}$ in DL.

Holot. $\delta$ on a pointed card, a leg on the same card, dissected parts on another card, "Iguazú / Misiones. Arg. / Dr. Duret XI-45” handwr.; "1266" handwr. with green ink; "Holotypus" print. on red paper, black frame; "Platytylus / dureti n. sp. [handwr.] / J.C.M. Carvalho det. 1988 [print.]" on white paper, black frame.

ecuadorianus [Adpiasus]. Carvalho \& D.L. Carpintero 1986: 291-292, f. 1-4. Ecuador: Quevedo V-1978 leg. Fritz, holot. $\widehat{o}$ in DL.

Holot. $\delta$ on a pointed card, dissected parts on another card, "Quevedo / Ecuador / Fritz col. V-76" handwr.; "947" typed with red ink;
"Adpiasus / ecuadorensis (sic) / n. sp. [handwr.] / det. J.C.M. Carvalho 19_[print.]" on white paper, black frame.

Note: The specific epithet differs slightly from the published one, ecuadorensis against ecuadorianus, but oherwise the labels seem authentic.

fratruelis [Capsus (Deraeocoris)]. Berg, 1880: 17. Buenos Aires Prov.: Río Colorado leg. Doering, one $\hat{\partial}$, one $q$.

Neot. missing on a pointed card, broken parts on the same card, "Argentina - Santa Fe / D ${ }^{\circ}$ Garay - Col. Mascías / M.J. Viana XI.1942" print., black frame; "Hyporminocoris / fratruelis / (Berg, 1878) / Det. Carpintero" handwr.; "Neotypus" print. on red paper.

Notes: The specimen got detached from the card and is lost. Currently in genus Hyporminocoris.

iguazuensis [Prepops]. Carvalho \& D.L.Carpintero 1987: 27，f. 19-22. Misiones Prov.: Iguazú National Park XII.1979 holot. $\delta$ in DL, parat. $\delta$ in CJC.

Holot. $\delta$ on a card, "P.N. Iguazu / Misiones / XII-79" handwr.; "Holotypus" print. on red paper; "403" typed with red ink; "Prepops / iguazuensis n. sp. [handwr.] / det. J.C.M. Carvalho 19 [print.]" on white paper, black frame.

rionegrensis [Hadronemella]. Carvalho \& D.L.Carpintero 1991: 771, f. 25-28. Río Negro Prov.: Coronel Gomes (sic, pro Gómez) XI.1945, holot. $\delta$ in DL.

Holot. $\delta$ on a pointed card, dissected parts on another card, "Cnel. Gomez / Rio Negro / XI45" handwr.; "304" handwr. with green ink; "Holotypus" print. on red paper, remainings of a black frame; "Hadronemella / rionegrensis $\mathrm{n}$. sp. / C.\&.C. [handwr.] / J.C.M. Carvalho det. 1988 [print.]" on white paper, black frame.

rodeana [Nanniresthenia]. Carvalho \& D.L. Carpintero 1989: 1117-1119, f. 27. Catamarca Prov.: Rodeo, leg. Golbach I.1958, holot. ${ }^{+}$in DL.

Holot. + on a pointed card, "El Rodeo / Catamarca / I..58 Arg. / Golbach" handwr.; "1286" handwr. with green ink; "Holotypus" print. on red paper; "Nanniresthenia / rodeana / n. sp. / Det. Carpintero" handwr. on white paper, black frame.

sanluisensis [Hadronemella]. Carvalho \& D.L. Carpintero 1991: 771-773, f. 29-33. San Luis Prov.: San Gerónimo, leg. Viana I.1972, holot. 
$\hat{~}$, parat. six $\hat{\rho}$, three $q$ in DL and CJC. Holot. $\hat{\sigma}$ on a card, "S. Geronimo / San Luis / I-72 Viana" handwr., remainings of a black frame; " 344 " handwr. with green ink; "Holotypus" print. on red paper, remainings of a black frame; "Hadronemella / sanluisensis / $\mathrm{n}$. sp. / Det. Carpintero", handwr. on white paper, black frame.

tibialis [Iguazucoris]. Carvalho \& D.L. Carpintero 1985: 508, f. 1-5. Misiones Prov.: Iguazú National Park X.1980, holot. ô in DL, one parat. $\delta$ in CJC.

Holot. $\delta$ on a card, "P.N. Iguazu / Misiones / X-80" handwr.; "90" typed with red ink; "Holotypus" print. on red paper, remainings of a black frame; "Iguazucoris / tibialis n. sp. [handwr.] / det. J.C.M. Carvalho 19_ [print.]" on white paper, black frame.

vianai [Orthotylus]. Carvalho \& D.L. Carpintero 1991: 776-778, f. 42-45. San Luis Prov.: San Gerónimo, leg. Viana_I.1972, holot. $\widehat{\partial}$, parat. eight $\hat{\sigma}$, eight $q$ in DL and CJC.

Holot. $\widehat{\delta}$ on a rectangular card, "S. Gerónimo / San Luis. Arg. / I-72 Viana" handwr.; "360" handwr. with green ink; "Holotypus" print. on red paper, remainings of a black frame; "Orthotylus / vianai / n. sp. / Det. Carpintero" handwr. on white paper, black frame.

willineri [Chiloxionotus]. Carvalho \& D.L. Carpintero 1989: 1113-1116, f. 14-17. Tucumán Prov. $4300 \mathrm{~m}$ alt., holot. $\widehat{\delta}$ in DL.

Holot. $\delta$ on a pointed card, dissected parts on another card, "Tucuman / $4300 \mathrm{~m}$ Arg. / XI64" handwr.; "1287" handwr. with green ink; "Holotypus" print. on red paper, remainings of a black frame; "Chiloxionotus / willineri $\mathrm{n}$. sp. [handwr.] / J.C.M. Carvalho det. 1988 [print.]" on white paper, remainings of a black frame.

\section{Naucoridae}

asper [Limnocoris]. Nieser \& López Ruf 2001: 266, 301-302, 318, f. 103, 105-108, 157, 158. Brasil: Minas Gerais, bacia do Rio Doce: Rib. Indaía, VIII.1993, holot. $q$ brachypter., allot. $\delta^{\lambda}$ in IBH; parat. four $\hat{O}$, ten $\odot$ in several repositories, MACN not mentioned.

Two parat. metallized for scanning electron microscopy, glued on their backs to cards. One of them: "Brasil: MG / Bacia do / Rio Doce / L. Rocha", and “ \pm 1100 m Ri- / bão Indriá VIII.1993" xerocop. on blue paper. The other one: "O?" print.; "Brasil: M.G. / Sa. do Cipó / VIII.1993 / leg.: L. Rocha catchment / basin R. Doce / Rbão Indria / $1100 \mathrm{~m}$ asl." print.; "Limnocoris / asper Nie- / ser \& L. Ruf / paratype q" xerocop. on blue paper.

espinolai [Limnocoris]. Nieser \& López Ruf 2001: 266, 305-308, 318, f. 117-121, 123, 124, 167, 168. Brasil: Minas Gerais: Serra do Caraça: Santa Bárbara 12.X.1972, holot. \& brachypt., allot. $\delta$ in IBH, three parat. in other repositories, MACN not mentioned.

One parat. + metallized for scanning electron microscopy, glued on its back to a card, legs on another card, "Serra do Caraça / Santa Bárbara / Minas Gerais / Brasil" print.; "H.N. Espínola col. / Data [print.] 21/10/72 [handwr.]"; "Limnocoris / espinolai / Nsr. \& LR / paratype ᄋ" xerocop. on blue paper.

kolla [Ambrysus (Ambrysus)]. López Ruf 2004: 98-101, f. 1-4. Tucumán Prov.: La Hoyada, holot. , two parat. in IML. Jujuy Prov.: Arroyo Los Berros, parat. three $\delta$, four $\circ$. Other parat. Tucumán Prov.: Las Juntas; La Rioja Prov.: near Chepes.

One parat. pinned, "Tucumán / La Hoyada / 23.II.49 / leg. E. Domínguez " handwr., black frame. One parat. pinned, "Jujuy / Ruta 34 / A ${ }^{\circ}$ Los Berros ", "9-XI-1992 / Leg. López Ruf" print., black frame. Both: "Paratipo" handwr. on green paper; "Museo de La Plata / Ambrysus (Ambrysus) / kolla López Ruf / Det.: López Ruf “ print. on white paper, black frame.

lanemeloi [Limnocoris]. Nieser \& López Ruf 2001: 303, 309-311, 319, f. 130-133, 141, 175, 176. Brasil: Minas Gerais: Rio do Peixe 27.III.1996, holot. $\odot$ brachypt., allot. $\delta$ in IBH, parat. in different repositories, MACN not mentioned.

Two parat., one pinned, one metallized for scanning electron microscopy, glued on its back on a card, "”, “ ‘” print.; "Brasil: M.G. / S. Roque de / M. 27.III.96 / leg. Nieser / N9636 Rio do / Peixe" xerocop. from typed, on blue paper; "Limnocoris / lanemeloi / Nsr. \& LR. / paratype 우 [ภ]" print. on blue paper.

porphyros [Limnocoris]. Nieser \& López Ruf. 2001: 314-316, 319, f. 143, 146-150, 191, 192. Brasil: Minas Gerais: bacia do Rio Doce, ribeir. Indaía VIII.1993, holot. $\sigma^{\top}$ brachypt., allot. $q$ in IBH, parat. nine ${ }^{\lambda}$, five $\rho$ in several repositories, MACN not mentioned.

Two parat. metallized for scanning electron microscopy, glued on their backs to cards. One of 
them "O", print., "Brasil: MG / Bacia de / Rio Doce / L. Rocha" ," \pm 1100 m ri- / bão Indriá / VIII 1993" xerocop. from typed; "Limnocoris / porphyros / Nsr. \& L.- R. / paratype ${ }^{\prime}$ ” xerocop. from typed, on blue paper. The other one " $\mathrm{O}$ " print.; "Brasil: MG / Sa. do Cipó / VIII.1993 / leg. L. Rocha / catchment / basin R. Doce / Rbão Indria / $1100 \mathrm{~m}$ asl" xerocop. from typed; "Limnocoris / porphyros / Nsr. \& L.-Ruf / paratype ${ }^{\lambda}$ " xerocop. from typed, on blue paper.

surinamensis [Limnocoris fittkaui subsp.]. Nieser 1975: 70-72, pl. 4b, f. 87-91. Surinam; Saramacca, holot. $\delta^{\lambda}$, allot. + , parat. five $\delta^{\lambda}$, four $q$ in ZMU, other parat. in AMNH, BM, KU, MO, MS, MACN, MLE, RL, USNM. The spec. were preserved in ethanol, then dried and pinned.

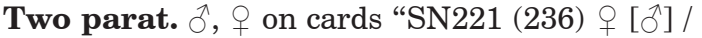
paratype / Limnocoris / fittkaui / suri / namensis [Lim / nocoris fitt / kaui surina / mensis]" handwr.; "Suriname Saramacca / Troelikreek-system / 4-XI-1969 [18-XI-1969] SN221 [SN236] / ...gt N. Nieser" print. I add "Paratypus" print. on yellowish orange paper, black frame.

\section{Notonectidae}

inca [Notonecta (Paranecta)]. Mazzucconi 2000b: 32-34, f. 1, 3, 5, 6, 8, 10, 12, 14, 16-18. Perú: Junín: Concepción. 3260 m, Woytkowski leg., holot. $\sigma^{\lambda}$, allot. + , sixty parat. in SEM; six parat. in MACN.

Four parat. (two $\hat{\sigma}$ with plastic microvials with genit.; two 9 ) pinned "Peru S.A., April 1315 [13-15, 7-8, 7-8] 1935, F. Woytkowski / near Concepción; Andes 3260 meters [above sea] Rio Mantaro [near or in Rio Mantaro] "; "Field notes $\mathrm{N}^{\circ} 3514$ [3514, 358, 358]". Two parat. $\hat{\delta}$,, , pinned, each with a plastic microvial with gen$i t$., the $q$ also with the tibia + tarsus of a hind leg, "Peru Concepción pools by Rio Mantaro, 3260 m. 7-10 April 1935, Woytkowski". All six: "Paratypus" print. on yellow paper, black frame; "Notonecta / (Paranecta) inca / S.A. Mazzucconi" handwr. on white paper.

\section{Reduviidae}

abbas [Brontostoma]. D.J. Carpintero 1980: 1112, f. 15. Venezuela: Portuguesa: Acarigua, leg. Carcavallo, holot. $\sigma^{\lambda}$ in CC.

Holot. $\delta$ pinned, genit. glued to a transparent card on another pin, "Holotypus 0 " print. on red paper, remainings of a black frame, "Venezuela
/ Portuguesa / Carcavallo / 1973" handwr., red frame; "Colección / Dr. Carpintero / Argentina" print., black frame; "Brontostoma / abbas / nov. sp. [handwr.] / Det. Dr. Carpintero [print.]" on white paper, black frame. On the pin with genit.: "Brontostoma / abbas [handwr.] / Det. Dr. Carpintero [print.]", black frame.

albidops [Pseudodaraxa]. D.J. Carpintero 1980: 19, f. 30. Entre Ríos Prov.: El Palmar National Park, holot. ô in CC.

Holot. Oे on a transparent card, "Holotypus $\delta^{\text {T" }}$ print. on red paper, black frame; "El Palmar / E. Ríos / Dic. 1973" handwr., remainings of a black frame; "Colección / Dr. Carpintero / Argentina" print., black frame; "Psudodaraxa / albidops / nov. sp. [handwr.] / Det. Dr. Carpintero [print.]", on white paper, black frame.

annulipes [Daraxa (Daraxacera)]. D.J. Carpintero 1980: 18, f. 18. Córdoba Prov.: La Serranita, holot. $\delta$ in CC. Tucumán Prov., allot. $q$ in MACN.

Holot. $\delta$ on a transparent card, "Holotypus $\lambda$ " print. on red paper, remainings of a black frame; "Anisacate / Córdoba / VII-1973 HN / Carpintero" handwr.; "Colección / Dr. Carpintero / Argentina" print., black frame; "Daraxa / (Daraxacera) / annulipes / nov. sp. [handwr.] / Det. Dr. Carpintero [print.]"on white paper, black frame.

Notes: The allot. was recorded in the main catalog (Bachmann 1999: 211). The locality La Serranita lies in Anisacate, Córdoba Prov.

arlequin [Brontostoma]. D.J. Carpintero 1980: 11, f. 14. Venezuela: Portuguesa: Acarigua, holot. $\delta$ in CC.

Holot. 0 pinned, genit. glued to a transparent card, on another pin, "Holotypus ${ }^{\text {t" }}$ " print. on red paper, black frame; "Venezuela / Acarigua / Portuguesa / Carcavallo 76" handwr., red frame; "Colección / Dr. Carpintero / Argentina" print., black frame; "Brontostoma / arlequin / nov. sp. [handwr.] / Det. Dr. Carpintero [print.]" on white paper, black frame.

australis [Daraxa]. D.J. Carpintero 1980: 15, f. 21. Buenos Aires Prov.: Glew, in CC.

One synt. + on a transparent card, "Holotypus Q" print. on red paper, black frame; "Glew / Prov. Bs. As. / Dic. 1974" handwr., black frame; "Daraxa / (Daraxa) / australis / nov. sp. [handwr.] / Det. Dr. Carpintero [print.]" on white paper, black frame.

Note: No type was formally designated. 
bolivari [Triatoma]. Carcavallo, Martínez \& Peláez 1987: 476-477, f. 1, 2. México: Colima, highway from Minotitlan to Santiago, San José Stream, holot. ${ }^{\lambda}$, twenty-three parat. Nayarit: Compostela, ten parat. Jalisco: Chamela, seven parat. in several repositories, including MACN. One parat. on a card, "México / Jalisco / Chamela / Est. Biol. UNAM / coll. Martínez / AG.1984" handwr.; "Paratipo" print. on grayish green paper; "Triatoma / bolivari ô sp. nov. / R.U. Carcavallo y [handwr.] / A. Martínez det. 1984 [print.]" on reddish orange paper, black frame.

boliviana [Pothea]. D.J. Carpintero 1980: 4, f. 1. Bolivia: Chapare: Siberia, holot. + in CC, one parat. $q$ in $\mathbf{C M}$.

Holot. $P$ on a transparent card, "Holotypus / P.b.1" print. on red paper, black frame; "Bolivia Chapare/SIBERIA I.72/ Fritz col." handwr., black frame; "Colección / Dr. Carpintero / Argentina" print., black frame; "Pothea / (Pothea) / boliviana / 9 [handwr.] / Det. Dr. Carpintero [print.]", black frame.

Note: One parat. was recorded in the main catalog (Bachmann 1999: 212).

breddini [Daraxa (Daraxa)]. D.J. Carpintero 1980: 15, f. 20. Salta Prov.: Pocitos, holot. ${ }^{1}$ in CC.

Holot. $\widehat{\delta}$ on a transparent card, "Holotypus ${ }^{\lambda}$ " print. on red paper, black frame; "Arg.-Salta / Pocitos 12-71 / Fritz col." handwr.; "Colección / Dr. Carpintero / Argentina" print., black frame; "Daraxa / (Daraxa) / breddini / nov. sp. [handwr.] / Det. Dr. Carpintero [print.]" on white paper, black frame.

Note: There is a further spec., labeled "Paratypus 7", not mentioned in the original description, see at the third list, below.

castaneum (sub castaneus) [Brontostoma]. D.J. Carpintero 1980: 10-11, f. 13. Misiones Prov.: Iguazú National Park, holot. $\delta$ in MACN. Paraguay, one parat. $\delta$ in CM.

Holot. $\delta$ pinned, genit. glued to a transparent card, on another pin, "Holotypus ${ }^{\lambda}$ " print. on red paper, remainings of a black frame; "P.N. Iguazú / Misiones / XII.1979" handwr.; "Colección / Dr. Carpintero / Argentina" print., black frame; "Brontostoma / castaneus / nov. sp. [handwr.] / Det. Dr. Carpintero [print.]" on white paper, black frame.

Notes: The specific epithet should be spelled castaneum (gender neuter). One parat. was recorded in the main catalog (Bachmann 1999: 212, 221); the holot. was then stated as 'not found'. dureti [Rhiginia]. D.J. Carpintero 1980: 26, f. 37. Ecuador: Esmeraldas: San Lorenzo, holot. $\delta$ in CC, two parat. $\delta$ in CM, one parat. in ISM.

Holot. $\delta$ on a transparent card, dissected parts on another card, "Holotypus ${ }^{\prime}$ " print. on red paper, black frame; "Ecuador / Esmeralda / Duret col. / X-1948 / San Lorenzo" handwr., red frame; "Colección / Dr. Carpintero / Argentina" print., black frame; "Rhiginia sp. [handwr.] / Wygodzinsky det. [print.]"; "Rhiginia / dureti / nov. sp. [handwr.] / Det. Dr. Carpintero [print.]" on white paper, black frame.

Note: Two parat. were recorded in the main catalog (Bachmann 1999: 213).

fritzi [Pothea (Pothea)]. D.J. Carpintero 1980: 5-6, f. 4. Bolivia: Chapare: Yungas, Fritz. leg., holot. $\delta$ in CC, eight parat. $\delta$ in CM.

Holot. $\delta$ on a transparent card, "Holotypus / P.f. 1" print. on red paper, black frame; "Bolivia Chapare / Siberia 1-72 / Fritz col." handwr., black frame; "Colección / Dr. Carpintero / Argentina" print., black frame; "Pothea / (Pothea) / fritzi / $\sigma^{\lambda}$ [handwr.] / Det. Dr. Carpintero [print.]" on white paper, black frame.

Note: Four parat. were recorded in the main catalog (Bachmann 1999: 213-214).

funerea [Triatoma]. D.J. Carpintero \& Leguizamón 1994: 111-115, f. 1, 2, 5, 7, 9-13, 15-20, photos 1-6. Misiones Prov.: Dep. San Ignacio, Col. Santa Rosa X.1987, holot. ㅇ, which was reared, laid eggs, the nymphs born were also reared and one $\delta$ and thirteen nymphs were finally obtained and designated as parat. Holot. + , three parat. in MACN, other parat. in CC and BM.

Holot. $q$ pinned, right fore wing extended, "Holotypus [print.] / T. funerea / DJC.MNL [handwr.]" on red paper, black frame; "Argentina [print.] / Misiones / S. Ignacio / C ${ }^{\circ}$ S. Rosa [handwr.]", black frame; "Triatoma / funerea / nov. sp. / Carpintero y / Leguizamon / mayo 1992" handwr., black frame. One parat. $\delta$ pinned, genit. on a cover glass on the same pin, "Paratipo / $\mathrm{N}^{\circ} 1 / \mathrm{AD}$ ' " typed on yellow paper, remainings of a gray frame; "Triatoma / funerea / nov. sp. / Carpintero y Leguizamón / mayo 1992" handwr. on white paper, black frame. A nymphal exuvia, pinned, three nymphs of different instars and an egg glued on transparent cards, on separate pins. The egg: "Huevo / T. funerea" typed. The nymphs "Paratipo / $\mathrm{N}^{\circ} 2 / \mathrm{N} \mathrm{V}^{\circ}\left[\mathrm{N}^{\circ} 4 / \mathrm{N}\right.$ $\left.\mathrm{IV}^{\circ}, \mathrm{N}^{\circ} 9 / \mathrm{N} \mathrm{II}^{\circ}, \mathrm{N}^{\circ} 11 / \mathrm{N} \mathrm{I}^{\circ}\right]$ " typed on yellow- 
ish paper, gray frame. All: "Argentina / Misiones / S. Ignacio / Co S. Rosa X-89 [handwr.]”, black frame; "Triatoma / funerea / nov. sp./ Carpintero y Leguizamón / mayo 1992" handwr. on white paper, black frame.

Note: Synonymized with Triatoma melanosoma Martínez, Olmedo \& Carcavallo 1987 by Lent, Jurberg \& Galvão (1995), and by Carcavallo, Galvão \& Lent (1998).

gallardoi [Triatoma]. D.J. Carpintero 1986: 114-119, f. 1-6, photos 1-5, map 1. Entre Ríos Prov.: San José de Feliciano, holot. $\widehat{o}$ in CC.

Holot. $\delta$ on a transparent card, genit. on a cover glass on another pin, "Holotypus" print. on red paper; "República Argentina / Provincia Entre Ríos / San José de Feliciano / Dic. 1972 Carpintero col." handwr., black frame; "Colección / Dr. Carpintero / Argentina" print., black frame; "Triatoma / gallardoi [handwr., label somewhat damaged] / nov. sp. [handwr.] / Det. Dr. Carpintero [print.]" on white paper, black frame.

guttata [Rhiginia]. D.J. Carpintero 1980: 26-27, f. 38. Jujuy Prov.: Yuto, holot. $\odot$ in CC. Bolivia: Beni, allot. $\partial^{\lambda}$ ex coll. Berg, at MACN. Bolivia two parat. $\delta$. Argentina one parat. $\delta$, all in $\mathbf{C M}$.

Holot. $\&$ pinned, "Holotypus $\odot "$ print. on red paper, remainings of a black frame; "Colección / Dr. Carpintero / Argentina” print., black frame; "Rhiginia guttata / nov. sp. [handwr.] / Det. Dr. Carpintero [print.]" on white paper, black frame.

Notes: The allot. $\delta$ and two parat. $\delta$ were recorded in the main catalog (Bachmann 1999: 214). A synonym of Rhiginia corrugata Maldonado Capriles 1972, fide D.J. Carpintero, personal communication VII.1994.

lilloi [Brontostoma]. D.J. Carpintero 1980: 10, f. 12. Tucumán Prov.: Horco Molle, holot. $\delta$ in CC. Bolivia, one parat. $\delta$ in CM.

Holot. $\delta$ pinned, a leg on a card, genit. on a transparent card, on another pin, "Brontostoma / lilloi [handwr.] / Det. Dr. Carpintero [print.] “, black frame; "Holotypus ${ }^{\top}$ " print. on red paper, black frame; "Tucuman / Horco Molle / mayo 1972" handwr.; "Colección / Dr. Carpintero / Argentina" print., black frame; "Brontostoma / lilloi / nov. sp . [handwr.] / Det. Dr. Carpintero [print.]" on white paper, black frame; "Brontostoma / sp. [handwr.] / Wygodzinsky det. [print.]”, remainings of a black frame.

Note: A parat. was recorded in the main catalog (Bachmann 1999: 215). littoralis [Pseudodaraxa]. D.J. Carpintero 1980: 20, f. 31. Entre Ríos Prov.: Concordia, holot. $\delta$, two parat. $\delta$ in CC; eight parat. $\delta$ in MACN.

Holot. $\widehat{\delta}$ on a transparent card, "Holotypus $\widehat{\phi}$ " print. on red paper, remainings of a black frame; "De trampa / luz / Col. S. Rosa / 8-4-1974 / N³6 [handwr., obverse] / Estación / Experimental / de Concordia / Entre Ríos / Argentina [print., reverse]", black frame; "Colección / Dr. Carpintero / Argentina" print., black frame; "Pseudodaraxa / littoralis / nov. sp. [handwr.] / Det. Dr. Carpintero [ print.]" on white paper, black frame.

Note: Eight parat. were recorded in the main catalog (Bachmann 1999: 215).

melanosoma [Triatoma infestans ssp.]. Martínez, Olmedo \& Carcavallo 1987: Misiones, dep. San Ignacio: Colonia Santa Rosa, leg. Olmedo. Holot. $\delta$, allot. + , parat. 15 , 15 , 15 nymphs of each stage, in CM.

Four parat. (two pinned, two on pointed cards) "Argentina / Misiones / Oberá / Col. Sta. Rosa [Sta. Rosa] - Olmedo / Febr.982" handwr. by Martínez; "Paratipo" print. on yellowish orange paper; "Triatoma / infestans / melanosoma / Carcavallo y [handwr.] / A. Martínez det. 1987 [print.]" on yellowish orange paper. One parat.? pinned, "PARATIPO" handwr. on bright red paper; "Argentina / Misiones / Posadas / H. Martínez leg / Abr. 1987" handwr. on green paper; "Triatoma infestans / melanosomica (sic) / Hemiptera / Reduviidae" handwr. on white paper.

Notes: The spec. from Posadas is dubiously a paratype, as it was not originally mentioned. There is another $\hat{o}$ specimen, not labeled as a type, with a glass vial, from Posadas, H. Martínez leg.

misionensis [Bactrodes]. Coscarón \& Melo 2003: 5, 11-13, f. 3M-V, 4L. Brasil: Goiás: Goiana, holot. $\delta$ in CAS. Misiones Prov.: Concepción: Santa María, 48059, three parat. $\delta$ in MACN. Other parat. in AMNH and CAS.

Five parat., two of them on pointed cards (one with a plastic microvial), three of them on cards on one pin, "Misiones - Argentina / Dep. Concep. Sta María / M.J. Viana" print., black frame, one of them also "XII.46" handwr.; two of them "48059" handwr., red frame. All: "Bactrodes / misionensis / Coscarón det." handwr. on white paper, black frame; "Paratipo" print. on red paper.

Note: The entry 48059 in the registration book of the Entomology Division refers to three reduviids from Misiones Prov.: Santa María, M.J.Viana leg. XII.1946. 
orientalis [Cricetopareis tucumana ssp.]. D.J. Carpintero 1980: 29-30, f. 41, 42. Buenos Aires Prov.: Otamendi, holot. in CC; Buenos Aires Prov.: Las Flores, Daguerre leg., allot. + in MACN;

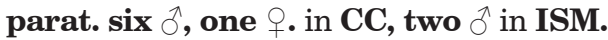

Holot. $\widehat{o}$ pinned, genit. in balsam on a transparent card, on another pin, "Holotypus $\delta^{\lambda "}$ print. on red paper, black frame; "Otamendi [handwr.]/ Pcia. Bs. As. [print.] / XII-1964 [handwr.]", black frame; "Colección / Dr. Carpintero / Argentina" print., black frame; "Cricetopareis / tucumana / orientalis / n. s. [handwr.] / Det. Dr. Carpintero [print.]" on white paper, black frame. On the pin with genit.: "Cricetopareis / tucumana / orientalis / Tipo [handwr.] / Det. Dr. Carpintero [print.]" on white paper, black frame. Eight parat. pinned: one of them, $q$ Misiones Prov.; three of them, đิ Entre Ríos Prov.: El Palmar; one of them $\delta$ E. Ríos Prov.: Villa Elisa; two of them $\delta^{\lambda}$ E. Ríos Prov: Primero de Mayo; one of them $\delta$ E. Ríos Prov.: Pronunciamiento.

Notes: The allot. ㅇ was recorded in the main catalog (Bachmann 1999: 216). Misiones was not mentioned.

paraguaya [Cricetopareis]. D.J. Carpintero 1980: 28-29, f. 40. Paraguay: Olimpo, holot. in CC. Paraguay: Cororó, leg. Viana, allot. $q$ in MACN. Paraguay, one parat. in CC, one $Q$ in MACN, two $\odot$ in CM, three $\odot$ in ISM. Misiones Prov., four $P$ in CM; one $\delta$ in ISM.

One parat. pinned, "Paratypus $\delta$ " print. on yellow paper, black frame; "Paraguay / Caá Guazú / Schade col. / IX-48" handwr., red frame; "Cricetopareis / paraguaya / N. S. [handwr.] / Det. Dr. Carpintero [print.]" on white paper.

Note: The allot. $q$ and five parat. were recorded in the main catalog (Bachmann 1999: 216).

paraguaya [Pothea (Pothea)]. D.J. Carpintero 1980: 7, f. 6. Paraguay: Itaguá (sic, pro Itapuá), leg. Carcavallo, holot. $\delta$ in CC.

Holot. $\delta$ on a transparent card, genit. on another transparent card, on another pin, "Holotypus $\lambda$ " print. on red paper, remainings of a black frame; "Itapuá - Vera / Paraguay / XII-54 RUC" handwr., glued to a card with a red frame; "Colección / Dr. Carpintero / Argentina" print., black frame; "Pothea / (Pothea) / paraguaya / nov. sp. [handwr.] / Det. Dr. Carpintero [print.]" on white paper, black frame.

zelichi [Daraxa (Daraxa)]. D.J. Carpintero 1980: 14, f. 19. Entre Ríos Prov.: Villa Elisa, holot 우 in $\mathbf{C C}$.
Holot. on a transparent card, "Holotypus $q "$ print. on red paper, remainings of a black frame; "Entre Ríos / Villa Elisa / Abril 1975 / cueva de / Edentado" handwr.; "Colección / Dr. Carpintero / Argentina" print., black frame; "Daraxa/ (Daraxa) / zelichi / n. sp. [handwr.] / Det. Dr. Carpintero [print.]" on white paper, black frame.

\section{Rhyparochromidae}

argentinensis [Erlacda]. Dellapé \& Melo 2004: 201-205, f. 1-11, tab 1. Corrientes Prov.: Ituzaingó, reserva Santa María, and several other localities in the province, holot. $\delta$ in MLP. The following in MACN: Salta Prov.: Caraparí Stream; Tucumán Prov.; Chaco Prov.; Catamarca Prov.: Concepción de Capayán; La Rioja Prov.: Ilias (sic, pro Iliar); Buenos Aires Prov.: Caseros, and Martín García Is., leg. Viana.

Nine parat. on pointed cards: One of them: "Caseros / XII.1946" handwr. One of them: "Isla M. García / 14-19-III-1932 / M.J.Viana col." print. One of them: "Chaco" print. Two of them: "La Rioja - Ilias (sic, pro Iliar) / Mateo Gómez / col." print. One of them: "Tucuman / 17-11-1951 / N. Kormilev col." print. One of them: "Salta - rio Canapari (sic, pro Caraparí) / 29-III-1948 / N. Kormilev col.", print. Two of them: "Catamarca- / Concepción de / Capayan / II-1958" print. All nine: "Erlacda / argentinensis / Dellapé \& Melo 2004" handwr. on white paper; "Paratipo" print. on red paper.

atricolor [Ischnodemus]. Berg 1892: 158-159. Few spec., San Luis Prov.; Uruguay: Rocha Dept., on Panicum prionitis.

Two synt. on cards, remounted, they show a pinhole, "Typus" print. with red ink on tiny labels, red frame; "Banda / Oriental" print. on green paper; "Ischnodemus / atricolor / Berg 1892" handwr., this label glued to the reverse of a card; "Ischnodemus / atricolor / Berg 1892 Typus [handwr.] / Det. Dr. Carpintero [print.]" on white paper.

Note: Banda Oriental is an old name of Uruguay.

bimaculatus [Pseudoparomius]. Dellapé \& Coscarón 2005: 6-7, f. 2, 10-14. Brasil: Pará: Belém, leg. Galiano, holot. in MACN. Parat.: Misiones Prov.: 17 de Octubre; Yacuí leg. A. Giai; Puerto Bemberg leg. Hayward; Puerto Aguirre leg. Hayward, in MACN. Bolivia: Caraveni, in MLP.

Holot. $\delta$ on a pointed card, a plastic microvial with dissected parts, "Brasil / Pará. Belém / 
VII.970 / M. Galiano" handwr.; "Holotipo" print. on red paper; "Pseudoparomius / bimaculatus / Dellapé \& Coscarón" print. on white paper, remainings of a black frame. Seven parat. on pointed cards, all seven: "Paratypus" print. on red paper; "Psudoparomius / bimaculatus / Dellapé \& Coscarón” print., remainings of a black frame. One of them: "Misiones - Pto / Bemberg / 17-30-III-1934 / Hayward" print. One of them: "Misiones Pto. / Aguirre / 1-6-III-1934 / Hayward" print. Three of them: "Misiones - Yacui / III-1950 / Giai" print. Two of them: "Misiones - Puerto / 17 de Octubre / 29-31-X1954 / De Carlo - Viana" print.

riojanus [Bergicoris]. Dellapé 2008: 102, 105, f. 3, 13-17. La Rioja Prov.: Illias (sic, pro Iliar), M. Gómez leg. holot. $\hat{O}$, parat. two $\hat{\partial}$, one $\stackrel{\circ}{\text {. in }}$ MACN; one $q$ in DL.

Holot. on a pointed card, a plastic microvial with dissected parts, "La Rioja - Ilias (sic, pro Iliar) / Mateo Gómez / col." print., black frame; "Holotipo" handwr. on red paper; "Bergicoris / riojanus / n. sp. / P. Dellapé det." handwr. Three parat. on pointed cards, (one of them with a plastic microvial), "La Rioja - Ilias (sic, pro Iliar) / Mateo Gómez / col." print., black frame; "Paratipo" print. on red paper, two of them also "Bergicoris / riojanus / n. sp. / P. Dellapé det."

Note: Bergicoris is a nomen nudum, as the decription lacks an explicit designation of type species; the specific epithet riojanus is available.

slateri [Pseudoparomius]. Dellapé \& Coscarón 2005; 9-11, f. 4, 24-28. Corrientes Prov.: Solari, holot., parat. Corrientes Prov.: Ituzaingó; San Roque, Bosq leg.; Uruguay: Punta del Este, parat., all in MLP. Buenos Aires, leg. Bruch; B. Aires Prov.: Punta Indio; Santa Fe Prov: Rosario; Villa Ana leg. Hayward; Uruguay: Atlántida; Cerro Chato, parat. all in MACN. B. Aires Prov.: Glew, parat. in DL.

Twelve parat. on pointed cards, eight parat. on rectangular cards, on 14 pins, all: "Paratypus" print. on red paper; "Pseudoparomius / slateri / Dellapé \& Coscarón" print. on white paper. Two of them: "Rca. Argentina / Prov. Buenos Aires / III.1896 / C. Bruch" print., date handwr., black frame. One of them: "R. Argentina/Buenos Aires / Coll. C. Bruch" print. One of them "Argentina / Bs. Aires [print.] / Punta Indio [handwr.]". Two of them on one pin "3967-8 / BA: Tornquist / Cerro Vacacuá 26 I 1947 / Leg. Bachmann Rossi" handwr. One of them: "Rosario" handwr. Two of them on one pin "Santa Fe / Villa
Ana (FCPSF) / 4/8-IX-1933 / Hayward" print. One + four of them: "Uruguay / 20-I-61 / C.S. Carbonell col." print. Two of them: "Atlántida" handwr. Two of them on one pin "Alto Alegre - Uruguay / 19-XII-1960 / C.S. Carbonell \& L. / Zolessi col." print. One of them: "Rio Cerro Chato / Uruguay 23-XI-59 / C.S. Carbonell, A. Mesa \& P. S. Martín / col." print. Four of them on one pin, "Rio Uruguay / Atlántida / I-1930 / A. Stevenin col." print.

\section{Saldidae}

penai [Pseudosaldula]. Schuh \& Polhemus 2009: 47-50, pl. 3, f. 1, 2, map 8. Salta Prov.: Santa Rosa de Tastil $3215 \mathrm{~m}$ alt., holot. $\delta$ in AMNH. Jujuy Prov: El Quemado and other localities; Salta Prov. Chile. Bolivia, many parat. $\hat{\alpha}$, $\rho$, in AMNH, MACN and other repositories.

Two parat. $\delta$, $\rho$ on pointed cards, "Argentina. Jujuy Prov. / El Quemado, below summit / Abra Potrerillo on Rt. 52 / $23^{\circ} 41.63 \mathrm{~S} 65^{\circ} 38.96$ W / 4170 m. Feb. 16, 1993 / R. T. Schuh, J.T. Polhemus / E. Domínguez" print.; "AMNH_ENT / AMNH_ENT 00023142" ["00023157"] print. Two parat. on pointed cards, "Argentina: Salta Prov.: / just NW of Santa Rosa de / Tastil on Rt.51 / $24^{\circ} 26.49 \mathrm{~S}, 65^{\circ} 58.01 \mathrm{~W}$, / 3215 m., Feb. 18, 1993 / R.T. Schuh, J.T. Polhemus" print. "AMNH ENT / AMNH_ENT 00023024 [00023003]" print. All: "Paratype / đ̊ [] Pseudosaldula / penai Schuh \& / Polhemus 2009" print. on sky blue paper.

pilosa [Pseudosaldula]. Schuh \& Polhemus 2009: 52-55, pl. 4, 7G, H, 8C-E, f. 1, 3, 14, map 10. Tucumán Prov.: Infiernillo, holot. in AMNH. Neuquen Prov.: San Martín de los Andes; Chubut Prov: $17 \mathrm{~km}$ of Esquel; Puelo Lake; Tucumán Prov.: near Tafí del Valle. Chile: Bio-Bio Prov., parat. in AMNH, MACN and other repositories.

Two parat. on pointed cards, "Argentina Tucuman / 15 km NW Tafi del Valle / 2800 m., Jan. 2-3, 1982 / R.T. Schuh and B.M. Massie / large permanent seep, / densely vegetated" print.; "AMNH_ENT / AMNH_ENT 00022907" ["00022908"] print. One parat. on a pointed card, "Argentina Tucumán, $28 \mathrm{~km} \mathrm{SE}$ of Amaicha / del Valle / 2800 m. Jan. 2, 1982" print.; "R.T. Schuh \& B.M. Massie / seep densely covered / with very short veget." print.; "AMNH_ENT / AMNH_ENT 00023500" print. Two parat. on pointed cards, "CHILE. Bio-Bio Prov. / $4 \mathrm{~km} \mathrm{E}$ of El Abanico, / 950 m. Nov 21,1981 / R.T Schuh \& N.I. Platnick" print.; "AMNH_ENT / AMNH_ 
ENT / 00022916 [00022917]" print. All five: "PARATYPE / ठै [o] Pseudosaldula / pilosa Schuh \& / Polhemus 2009" print. on sky blue paper.

vulgaris [Pseudosaldula]. Schuh \& Polhemus 2009: 61-66, f. 1, 18, 19, map 13. Perú: Ancash, holot. $\widehat{o}$ in AMNH. Perú: Amazonas, Ancash, Cusco, Huánuco, La Libertad, Cajamarca, parat. in AMNH, MACN and other repositories.

Three parat. on pointed cards, "PERU, La Libertad $12[14,14] \mathrm{km} / \mathrm{SW}$ of Huamachuco, Rio Bado / 3040 [3050, 3050] meters, Jan. 21, 1976 / R.T. \& J.C. Schuh, sandy river / margin, $10-70 \%$ veg. cover $\mathrm{N}^{\circ} 38$ [39, 39]" print.; "AMNH ENT / AMNH_ENT 00019919” [“00019717”, "00019983"] print. One parat. on a pointed card, "Perú: Cajamarca, $12 \mathrm{~km} \mathrm{~W}$ / of Llacanora, near / Cajamarca, 2350 meters, / Jan. 19, 1976 R.T. \& J.C. Schuh / sandy river margin, ( $\left.\mathrm{N}^{\circ} 22\right)$ " print., "AMNH_ENT / AMNH ENT 00019794" print. One parat. on a pointed card, "Peru. Cajamarca: $11 \mathrm{~km} \mathrm{~S} \mathrm{/} \mathrm{of} \mathrm{Cajabamba,} \mathrm{Jan.} \mathrm{20,} 1976$ / 2850 m., R.T. \& J.C. Schuh / steep sided roadside ditch / with moss and algal cover, / $\left(\mathrm{N}^{\circ} 31\right)$ " print.; "AMNH_ENT / AMNH_ENT 00020244" print. All five: "Paratype / $\partial^{\lambda}[\overline{0}]$ Pseudosaldula / vulgaris Schuh \& / Polhemus 2009" print. on sky blue paper.

\section{Veliidae}

anta [Paravelia]. Mazzucconi 2000a: 130-134, f. 3-19. Salta Prov.: Anta Dept., 50 km East of Las Lajitas, 23-31.I.1980, leg. Golbach, holot. o macropterous, allot. + . Paraguay: Dep. Concepción; Puerto Vallemí, VI.1952 leg. Bachmann, one parat. + , all in MACN.

Holot. $\delta$ on a card, a plastic microvial with dissected parts, allot. $\circ$, one parat. $\circ$ on cards, "Argentina / Prov. Salta / Dto. Anta, $50 \mathrm{Km} \mathrm{E}$ Las Lajitas / 23-31/1/1980 R. Golbach" handwr.; "Paravelia / anta / Mazzucconi" handwr. One parat. $\delta$ on a card, with a plastic microvial with dissected parts, body and genital capsule metallized for electron microscopy, dry mounted, "7064 / Paraguay / Dep. Concepción / Pto. Vallemi / VI.1952 / A.O. Bachmann" handwr., red frame.

\section{SPECIMENS LABELED AS TYPES BUT NOT PUBLISHED}

\section{Reduviidae}

sierrai [Sosius]. Maldonado Capriles \& D.J. Carpintero
One spec. on a pointed card, with only one leg, "Holotypus o" print. on red paper; "El Talar / Jujuy / abril 79" handwr.; "Colección / Dr. Carpintero / Argentina" print., black frame; "Sosius / sierrai / nov. sp. / Tipo [handwr.] / Det. Dr. Carpintero [print.]" on white paper, black frame.

Note: J. Maldonado Capriles and D.J. Carpintero (1993: 223) re-examined the spec., which is in a poor condition, setting doubt even about its inclusion in Sosius, and decided not to publish the supposed new species.

\section{Thyreocoridae}

\section{vaginatus [Thyreocoris]. Berg}

One spec. on a card, "Typus" print. with red ink, red frame; " 5453 " handwr., red frame; "Rep. Argentina / Gob. Formosa / 4.I.1908 / S. Venturi" print., date handwr., black frame; "Thyreocoris / vaginatus / Berg" handwr. on white paper, black frame. One spec. originally pinned, pin broken, later glued to a pointed card, "Typus" print. with red ink, red framed; " 5248 " handwr., red frame; "Rep. Argentina / Gob. Chaco / 20.XI.1897 / S. Venturi" print., date handwr., black frame; a rectangular green label without writing, black frame, reverse white; "Col. / Antigua" print. on grayish white paper, black frame; "[a picture of the Argentine flag] / tipo / saginatus [?vaginatus] / Berg" handwr. on white paper, green frame; folded; "Galgupha / (Psestophleps) / carbonatum [handwr.] / Det. / Kormilev 53 [print.]", black frame.

Notes: A “Typus", from Formosa Prov., was recorded in the main catalog (Bachmann 1999: 222). Currently in Galgupha.

\section{SPECIMENS LABELED AS TYPES BUT NOT MENTIONED IN THE ORIGINAL DESCRIPTION}

\section{Reduviidae}

breddini [Daraxa (Daraxa)]. D.J. Carpintero 1980: 15, f. 20. Salta Prov.: Pocitos, holot. of in CC.

One spec. pinned, "Argentina / Salta / Tartagal / Coll. Duret / II.44 / Tablillas" print. and handwr. One spec. pinned, "El Naranjo / Salta / R. Arg. / I.44" photographed from typed. Both: "Paratypus ${ }^{\lambda}$ " print. on yellow paper; "Daraxa / (Daraxa) / breddini / n. s. [handwr.] / Det. Dr. Carpintero [print.]" on white paper, black frame. 
Note: Only the holot., from Pocitos (Salta Prov.), was mentioned, see at the main list, above.

\section{CORRIGENDUM}

\section{Reduviidae}

andina [Pothea]. D.J. Carpintero \& J. Maldonado Capriles 1990: 452-453, f. 3, 6, 9, 12.

Note: This entry was erroneously referred to D.J. Carpintero 1990 in the main catalog (Bachmann 1999: 211).

\section{BIBLIOGRAPHY}

Bachmann, A.O. 1999. Catálogo de los tipos de Heteroptera (Insecta) conservados en el Museo Argentino de Ciencias Naturales. Revista del Museo Argentino de Ciencias Naturales n. ser. 1 (2): 191-230.

Berg, C. 1880. Hemiptera argentina. Ensayo de una monografía de los hemípteros heterópteros y homópteros de la República Argentina. Anales de la Sociedad Científica Argentina 9: 5-22.

Berg, C. 1881. Sinonimia y descripción de algunos hemípteros de Chile, del Brasil y de Bolivia. Anales de la Sociedad Científica Argentina 12: 259-272.

Berg, C. 1891 / 1892. Nova Hemiptera faunarum argentinae et uruguayensis. Anales de la Sociedad Científica Argentina 32, 1891: 164-175; 231-243; 277-287. 33, 1892: 5-11; 43-50; 65-72; 97-104: 151-165. 34, 1892: 82-96; 193-205.

Brailovsky, H. 1986. Revisión del género Scamurius Stal (Hemiptera - Heteroptera - Coreidae Discogastrini). Folia Entomologica Mexicana 70: 25-52, f. 1-54.

Brailovsky, H. 1989. Cuatro especies nuevas sudamericanas de la familia Largidae (Hemiptera Heteroptera). Anales del Instituto de Biología, Universidad Nacional Autónoma de México, ser. Zool. 60 (1): 77-90, pl. 1-3.

Brailovsky, H. 1995. Revisión del complejo "Cebrenis" (Hemiptera - Heteroptera - Coreidae - Coreinae Coreini). Publicaciones Especiales, Universidad Nacional Autónoma de México (15): 124 p., 196 f., cuadros.

Carcavallo, R.U., Galvão, C. \& Lent, H. 1998. Triatoma jurbergi sp. n. do Norte do Estado de Mato Grosso, Brasil (Hemiptera, Reduviidae, Triatominae) com uma atualização das sinonimias e outros táxons. Memorias do Instituto Oswaldo Cruz, Rio de Janeiro, Brasil 93 (4): 459-464, f. 1-7, 1 tab.

Carcavallo, R.U., Martínez, A. \& Peláez, D. 1987. Una nueva especie de Triatoma Laporte en México. Chagas 4: 476-477.

Carpintero, D.J. 1980. Nuevos Ectrichodiinae americanos (Insecta - Hemiptera - Reduviidae). Acta Scientifica, Inst. Entom. S. Miguel., ser. Entom. (14): 3-34, pl. 1, 2.

Carpintero, D.J. 1986. Aporte al conocimiento de los triatominos en la Argentina. Segunda comunicación. Revista del Museo Argentino de Ciencias Naturales Entomología 4 (5): 113-127, f. 1-6, photos 1-5, map 1, 1 tab.

Carpintero, D.J. \& Leguizamón, M.N. 1994. Triatoma funerea, nueva especie de Triatominae (Hemiptera - Reduviidae). CM, Publicación Médica 7 (3): 111121, f. 1-20, photos 1-6.

Carpintero, D.J. \& Maldonado Capriles, J. 1990. Contributions to the knowledge of American Ectrichodiinae. II. Notes about Rhiginia and Pothea (Hemiptera: Reduviidae). Journal of Agriculture, Puerto Rico 74 (4): 449-456, f. 1-16.

Carvalho, J.C.M \& Carpintero, D.L. 1985. Mirídeos neotropicais, CCLXVIII: Dois gêneros novos da República Argentina (Hemiptera). Anais da Academia Brasileira de Ciências 57 (4): 507-511, f. 1-7.

Carvalho, J.C.M \& Carpintero, D.L.1986. Mirídeos neotropicais, CCLXXIV: Descrições de quatro espécies da América do Sul (Hemiptera). Anais da Academia Brasileira de Ciências 58 (2): 291-296, f. 1-14.

Carvalho, J.C.M \& Carpintero, D.L.1987. Mirídeos neotropicais, CCLXXII: Descrições de sete espécies novas da tribo Resthenini Reuter (Hemiptera). Revista Brasileira de Zoologia 4 (1): 19-31, f. $1-26$.

Carvalho, J.C.M \& Carpintero, D.L.1989. Mirídeos neotropicais, CCCXI: Novas espécies da tribo Resthenini Reuter, colecionadas na Argentina e Peru (Hemiptera). Revista Brasileira de Biologia 49 (4): 1109-1123, f. 1-39.

Carvalho, J.C.M \& Carpintero, D.L.1991. Miridos neotropicales, CCCXXXVII: Descripción de doce especies nuevas de Orthotylinae (Hemiptera). Revista Brasileira de Biologia 51 (4): 763-780, f. 1-51.

Carvalho, J.C.M. \& Wallerstein, P. 1978. Mirídeos neotropicais, CCXVI: Descrições de seis espécies novas da República Argentina (Hemiptera). Revista Brasileira de Biologia 38 (3): 523-530, f. 1-25.

Coscarón, M.C. \& Melo, M.C. 2003. Revision of the subfamily Bactrodinae (Heteroptera, Reduviidae), with a phylogenetic analysis of Bactrodes. Zootaxa 304: 1-15, f. 1-4, tab. 1.

De Carlo, J.A, 1958. Identificación de las especies del género Horvathinia Montandon. Descripción de tres especies nuevas (Hemiptera - Belostomatidae). Revista de la Sociedad Entomológica Argentina 20, 1957 (3/4): 45-52, f. 1-26.

De Carlo, J.A. 1966. Un nuevo género, nuevas especies y referencias de otras poco conocidas de la familia Belostomatidae (Hemiptera). Revista de la Sociedad Entomológica Argentina 28, 1965: 97-109, pl. I, II.

Dellapé, P.M. 2008. Bergicoris, a new genus for Neotropical species previously placed in Cnemodus Herrich-Schaeffer, and a new species from Argentina (Heteroptera, Lygaeoidea, Rhyparochromidae). Deutsche Entomologische Zeitschrift 56 (1): 101107, f. 1-17, tab. 1.

Dellapé, P.M. \& Coscarón, M.C. 2005. Redescription of Pseudoparomius linearis (Stal), and description of 
three new species of Pseudoparomius Harrington (Rhyparochromidae: Heteroptera). Zootaxa 909: 1-12, f. 1-28.

Dellapé, P. M. \& Melo, M.C. 2004. A new species of Erlacda Signoret (Heteroptera: Lygaeoidea: Rhyparochromidae) from Argentina. Studies on Neotropical Fauna and Environment 39 (3): 201205.

Dellapé, P. M. \& Melo, M.C. 2007. Thaumastaneis nigricans, a new species of remarkable and mimetic Larginae (Hemiptera: Largidae) and the discovery of an ant-mimetic complex. Zootaxa 1475: 21-26, f. $1-11$.

Di Iorio, O., Turienzo, P., Masello, J. \& Carpintero, D.L. 2010. Insects found in birds' nests from Argentina. Cyanoliseus patagonus (Vieillot, 1818) [Aves: Psittacidae], with the description of Cyanolicimex patagonicus, gen. n., sp. n., and a key to the genera of Haematosiphoninae (Hemiptera: Cimicidae). Zootaxa 2728: 1-22, f. 1-27, tab. 1, 2, append. 1, 2.

Drake, C.J. \& Hottes, F.C. 1925. Four undescribed species of water-striders (Hemiptera, Gerridae). Ohio Journal of Sciences 25: 46-50.

Estévez, A.L. \& Schnack, J.A. 1977. Una nueva especie del género Gelastocoris Kirkaldy (Hemiptera, Gelastocoridae). Neotrópica, La Plata 23 (69): 8183, f. $1-8$.

Hungerford, H.B. 1923. Some studies on the genus Hydrometra in America North of Mexico with description of a new species (Hydrometridae, Hemip.). Canadian Entomologist 55 (3): 54-58, f. 1-15.

ICZN. International Commission on Zoological Nomenclature. 1999. International Code of Zoological Nomenclature 4th. ed.: xxix +306 p.

Lent, H., Jurberg, J. \& Galvão, C. 1995. Um sinônimo de Triatoma melanosoma Martinez, Olmedo \& Carcavallo 1987 (Hemiptera, Reduviidae). Entomología y Vectores 2: 81-82.

López Ruf, M.L. 2004. Una especie nueva del género Ambrysus (Hemiptera: Naucoridae). Revista de la
Sociedad Entomológica Argentina 63 (1/2): 97-101, f. $1-4$.

Maldonado Capriles, J. \& Carpintero, D.J. 1993. Redescription of the harpactorine genus Sosius Champion 1899, with the description of a new species (Heteroptera: Reduviidae). Proceedings of the Entomological Society of Washington 95 (2): 223227, f. 1-17.

Martínez, A., Olmedo, R.A. \& Carcavallo, R.U. 1987. Una nueva subespecie argentina de Triatoma infestans. Chagas 4 (1): 479-480 (on reprint: 7-8).

Mazzucconi, S.A. 2000a. Paravelia anta sp. n. and $P$. hungerfordi (Drake and Harris) from South America (Heteroptera: Veliidae). Aquatic Insects 22 (2): 129-137, f. 1-19.

Mazzucconi, S.A. 2000b. Notonecta (Paranecta) inca n. sp. from Perú (Heteroptera: Notonectidae). Journal of the Kansas Entomological Society 73 (1): 30-35, f. 1-18.

Nieser, N. 1975. The water bugs (Heteroptera: Nepomorpha) of the Guyana region. Studies on the Fauna of Suriname and Other Guyanas 16 (59): 1-310, f. 1-337, pl. 1-24.

Nieser, N. \& López Ruf, M.L. 2001. A review of Limnocoris Stål (Heteroptera: Naucoridae) in Southern South America East of the Andes. Tidschrift voor Entomologie 144: 261-328, f. 1-202.

Perez-Goodwyn, P. J. 2001. A new Hydrometra species from Argentina (Heteroptera: Hydrometridae). Florida Entomologist 84 (1): 127-130, f. 1-8.

Schnack, J.A. \& Estévez, A.L. 2005. On the taxonomic status of the genus Horvathinia Montandon (Hemiptera: Belostomatidae). Zootaxa 1016: 2127.

Schuh, R. \& Polhemus, J.T. 2009. Revision and analysis of Pseudosaldula Cobben (Insecta: Hemiptera: Saldidae): group with a classic Andean distribution. Bulletin of the American Museum of Natural History 32: 1-102, 31 f., 4 tab., 8 pl. 